Article

\title{
Dynamic Response of Electro-Mechanical Properties of Cement-Based Piezoelectric Composites
}

\author{
Yi Li ${ }^{1}$, Youwei Zhang ${ }^{2}$, Haiwei Dong ${ }^{1}$, Wenjie Cheng ${ }^{1}$, Chaoming Shi ${ }^{1}$ and Jiangying Chen ${ }^{1, *}$ \\ 1 Key Laboratory of Impact and Safety Engineering, Ningbo University, Ministry of Education, \\ Ningbo 315211, China; $2101090010 @$ nbu.edu.cn (Y.L.); 1801081010@nbu.edu.cn (H.D.); \\ Wenjie.Cheng@nottingham.edu.cn (W.C.); 2011081151@nbu.edu.cn (C.S.) \\ 2 Ningbo Rail Transit Group Co., Ltd., Ningbo 315101, China; qiumeifang@nbu.edu.cn \\ * Correspondence: chenjiangying@nbu.edu.cn; Tel.: +86-574-8760-0496
}

check for updates

Citation: Li, Y.; Zhang, Y.; Dong, H.; Cheng, W.; Shi, C.; Chen, J. Dynamic Response of Electro-Mechanical Properties of Cement-Based Piezoelectric Composites. Appl. Sci. 2021, 11, 11925. https://doi.org/ 10.3390/app112411925

Academic Editor: Doo-Yeol Yoo

Received: 24 November 2021 Accepted: 8 December 2021 Published: 15 December 2021

Publisher's Note: MDPI stays neutral with regard to jurisdictional claims in published maps and institutional affiliations.

Copyright: (c) 2021 by the authors. Licensee MDPI, Basel, Switzerland. This article is an open access article distributed under the terms and conditions of the Creative Commons Attribution (CC BY) license (https:// creativecommons.org/licenses/by/ $4.0 /)$.

\begin{abstract}
By employing ordinary Portland cement as a matrix and PZT-5H piezoelectric ceramic as the functional body, 1-3 and 2-2 cement-based piezoelectric composites were prepared. Quasistatic compression tests were performed along with dynamic impact loading tests to study the electro-mechanical response characteristics of 1-3 and 2-2 cement-based piezoelectric composites. The research results show that both composites exhibit strain rate effects under quasi-static compression and dynamic impact loading since they are strain-rate sensitive materials. The sensitivity of the two composites has a non-linear mutation point: in the quasi-static state, the sensitivity of 1-3 and 2-2 composites is 157 and $169 \mathrm{pC} / \mathrm{N}$, respectively; in the dynamic state, the respective sensitivity is 323 and $296 \mathrm{pC} / \mathrm{N}$. Although the sensitivity difference is not significant, the linear range of the 2-2 composite is $24.8 \%$ and $61.3 \%$ larger than that of the 1-3 composite under quasi-static compression and dynamic impact loading, respectively. Accordingly, the 2-2 composite exhibits certain advantages as a sensor material, irrespective of whether it is subjected to quasi-static or dynamic loading.
\end{abstract}

Keywords: electro-mechanical response; cement-based piezoelectric composites; impact loading; sensitivity

\section{Introduction}

Concrete structures have become omnipresent in our lives. During their service life, the combined action of environment and loads lead to easy damage in concrete structure [1-3], they are not only subjected to quasi-static loads, but also to various dynamic loads. Nowadays, elucidating the mechanical properties of concrete structures under dynamic loads has become one of the research hotspots in the civil engineering field $[4,5]$. Meanwhile, it has become a trend to rely on intelligent systems to implement health monitoring and damage forecasting methods for these structures [6-8]; however, it is difficult to practically apply these approaches [5-7]. Current piezoelectric smart materials exhibit several shortcomings, such as high costs, poor compatibility, and poor durability, which limit their applicability [5,8-10]. The incompatibility of their mechanical properties with those of embedded pressure sensors and matrixes tend to facilitate internal cracks. The large size of pressure components, incompatibility with concrete materials, and high costs also hinder the applicability of piezoelectric smart materials in the field of concrete structures [11-14].

Piezoelectric materials are widely used in structural damage diagnosis and health monitoring. Because of their low costs, fast response speeds, good reliability, suitable linear relationship, and decent electro-mechanical response characteristics, they also can be used in sensors and actuators [15-18]. In recent years, the good matching performance of cement-based piezoelectric composite materials and concrete materials has led to the development of a multifunctional sensor suitable for civil engineering $[8,11,19]$. Compared with traditional piezoelectric materials, this novel sensor has two outstanding advantages: good 
performance and designability, and better compatibility with the concrete structure [20]. Therefore, in the field of engineering structure design, the cement-based piezoelectric ceramic sensor embedded in the reinforced concrete structure has received continuous attention for monitoring internal dynamic stress [21,22].

According to the connection mode of the functional phase and matrix, cement-based piezoelectric composites can be categorized into more than ten models. Currently, three main composite materials are being researched and developed: 0-3, 1-3, and 2-2. Type 1-3 is a two-phase piezoelectric composite comprising a one-dimensional piezoelectric ceramic column, which is arranged in a three-dimensional connected cement matrix. Type 2-2 comprises a laminated piezoelectric ceramic phase and cement interlayer [23].

Qian et al. [24] researched the electrical domain switching of piezoelectric ceramics in cement-based piezoelectric composites under quasi-static and dynamic compression loads. They revealed that the loading stress increases with a rise in the electrical domain switching. Tang et al. [25] used a first-stage light gas gun to study the output electrical signals of PZT-5H under different loading rates. They demonstrate that at low loading rates, the output electrical signal of PZT-5H maintains a linear relationship with the applied load. At high loading rates, the efficiency associated with the conversion of kinetic energy to electrical energy for PZT-5H is low. Zhang et al. [26] used a Split Hopkinson pressure bar (Ningbo University, Noingbo, China) (SHPB) device to perform single and multiple impact compression tests on type 1-3 cement-based piezoelectric composites; they found that the material has a significant strain rate effect, while showing that it is a strain rate sensitive material. Meanwhile, the linear electro-mechanical response of the material has a critical point. Rong et al. [27] performed a dynamic analysis of cement-based piezoelectric composites by using the displacement method and found that the piezoelectric elastic theory is applicable to cement-based piezoelectric composites under different strain rates.

The existing research on the influence of quasi-static and dynamic impact loads on the electro-mechanical response characteristics of cement-based piezoelectric composites is mostly focused on type 0-3 and type 1-3, and there is a lack of comparative research on the electro-mechanical properties of cement-based piezoelectric composites with different structural types. In this study, quasi-static and dynamic impact compression tests were performed for type 1-3 and type 2-2 cement-based piezoelectric composites to study the electro-mechanical response characteristics under different load conditions. Then, the applicability and stability of sensor sensitivity were compared to provide theoretical guidance for the application of cement-based piezoelectric sensors in practical engineering.

\section{Materials and Methods}

\subsection{Sample Preparation}

Herein, the P.II 42.5 Portland cement paste with a water-cement ratio of 0.32 was used as the matrix, while PZT-5H piezoelectric ceramics were employed as the functional body and epoxy resin was applied to reinforce the interface bonding. Then, the cuttingcasting method was used to prepare type 1-3 and type 2-2 cement-based piezoelectric composites (the dimensions of type 1-3 and type 2-2 are $9 \mathrm{~mm} \times 9 \mathrm{~mm} \times 7 \mathrm{~mm}$ and $17 \mathrm{~mm}$ $\times 17 \mathrm{~mm} \times 8 \mathrm{~mm}$, respectively), as shown in Figure 1. Type 1-3 is a two-phase piezoelectric composite comprising a one-dimensional piezoelectric ceramic column arranged in a threedimensional connected cement matrix. Type 2-2 is composed of a laminated piezoelectric ceramic phase and cement interlayer. After cutting the samples, the cross-sectional size of the type 1-3 single ceramic column is $1 \mathrm{~mm} \times 1 \mathrm{~mm}$ (Figure 1a), and the cross-sectional size of a single type 2-2 ceramic body is $1 \mathrm{~mm} \times 15 \mathrm{~mm}$ (Figure $1 \mathrm{~b}$ ).

\subsection{Sample Packed}

During the compression experiment, the charge generated by the sample cannot be directly measured; therefore, the sample needs to be encapsulated. Subsequently, the samples were polished and cleaned, and a conductive silver paste was applied on the two electrode surfaces after air-drying. An aluminum foil has been used as the electrode 
material, and the insulating layer is made of a polyimide film. One side of the aluminum foil is bonded to the polyimide film, and the other side is bonded to the electrode surface of the sample by using a conductive silver glue. The diagram of this packaged structure is shown in Figure 2.

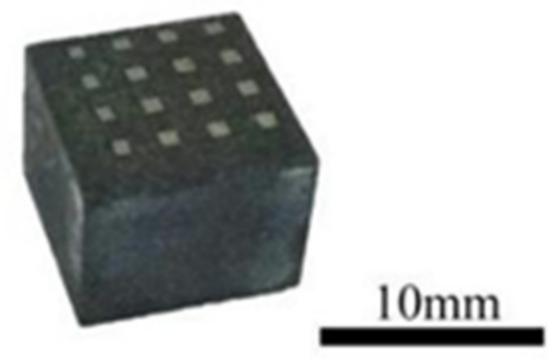

(a) type 1-3

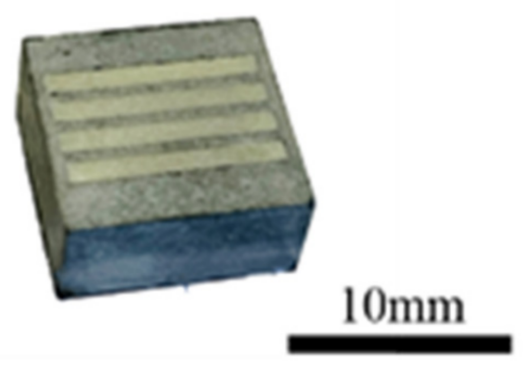

(b) type 2-2

Figure 1. Samples of cement-based piezoelectric composites.

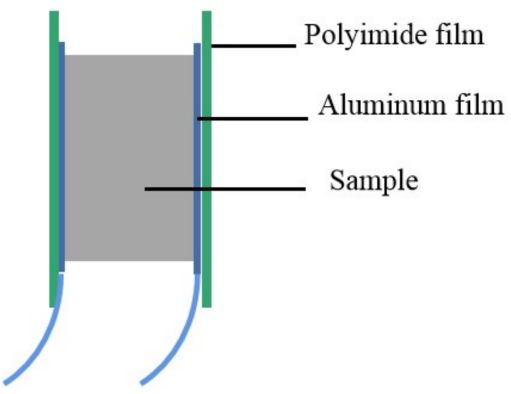

(a)

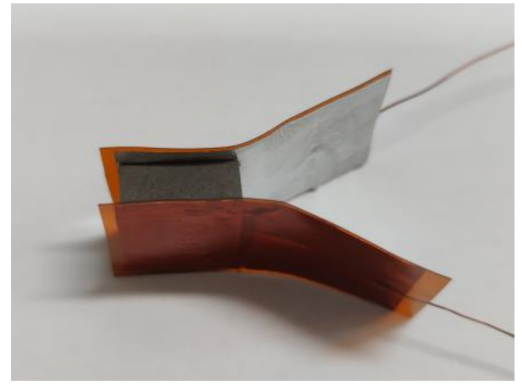

(b)

Figure 2. Packaged structure of the sample. (a) Schematic of the packaged structure; (b) Actual image of the packaged structure.

\subsection{Performance Test}

The Mechanical Testing System (MTS) is used to perform quasi-static compression tests on type 1-3 and type 2-2 cement-based piezoelectric composite samples. The alumina ceramic column is used to insulate the sample surface against the testing machine and output a high-impedance charge signal, which is converted into a low-output-impedance voltage signal via a charge amplifier; subsequently, the data signal is collected by the data collector and transmitted to the computer for processing, saving, and analyzing data. According to the cyclic loading characteristics, the initial stress value and loading frequency are controlled and the stress amplitude is increased sequentially; this causes the corresponding electric displacement to also increase. The initial stress value is 0 and the frequency is $1 \mathrm{~Hz}$. By determining the peak value of the electric displacement and fitting it into a straight line, the quasi-static sensitivity of the type 1-3 and type 2-2 cementbased piezoelectric composite samples can be obtained. The ratio of the output electric displacement $D(t)$ to the stress $\sigma(t)$ represents the static sensitivity of the sample.

An SHPB device is used to perform impact compression tests on type 1-3 and type 2-2 cement-based piezoelectric composites. The SHPB device are shown in Figure 3. The lengths of the impact bar, incident bar, transmission bar, and absorption bar are 400, 2000, 2000 , and $600 \mathrm{~mm}$, respectively; the bar diameter is $37 \mathrm{~mm}$, and the material used is highstrength spring steel. According to the one-dimensional stress wave and homogenization assumption of the SHPB technology, the stress $\sigma_{S}(t)$, strain $\varepsilon_{S}(t)$, strain rate $\dot{\varepsilon}_{S}(t)$ and average strain rate $\dot{\bar{\varepsilon}}$ of the sample can be calculated via Formulas (1)-(4) [28], respectively. 
Ordinary strain gauges are attached to both the incident and transmission bars to record the stress waveform in the bar during the test. To increase the rising edge time of the loading waveform and balance the internal stress of the sample, a brass wave shaper is attached to the front face of the incident bar.

$$
\begin{gathered}
\sigma_{S}(t)=\frac{E A}{A_{0}}\left[\varepsilon_{I}(t)+\varepsilon_{R}(t)\right] \\
\varepsilon_{S}(t)=\frac{2 c_{0}}{L_{0}} \int_{0}^{t}\left[\varepsilon_{I}(t)-\varepsilon_{T}(t)\right] d t \\
\dot{\varepsilon}_{S}(t)=\frac{2 c_{0}}{L_{0}}\left[\varepsilon_{I}(t)-\varepsilon_{T}(t)\right] \\
\dot{\bar{\varepsilon}}=\frac{\int_{0}^{\varepsilon} \dot{\mathcal{E}}_{S}(\varepsilon) d t}{\varepsilon_{S}}
\end{gathered}
$$

Striker Bar

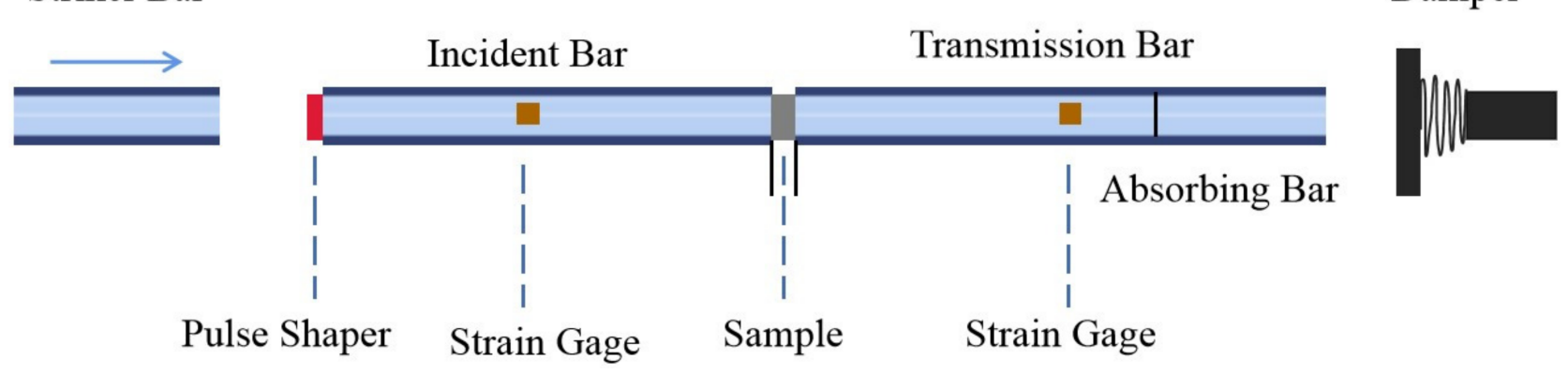

Figure 3. Schematic diagram of the SHPB device.

Here, $E$ is the elastic modulus of the steel bar, $A$ is the cross-sectional area of the steel bar, $C_{0}$ is the propagation speed of the stress wave signal in the steel bar, $A_{0}$ is the cross-sectional area of the sample, $L_{0}$ is the length of the sample, $\varepsilon_{I}(t)$ is the incident wave signal, $\varepsilon_{R}(t)$ is the reflected wave signal on the incident bar, and $\varepsilon_{T}(t)$ is the transmitted wave signal on the transmission bar.

\section{Results and Discussion}

\subsection{Quasi-Static Compression Test}

The quasi-static sensitivity calibration of the type 1-3 and type 2-2 cement-based piezoelectric composite was performed on the MTS universal testing machine by using compression tests. The quasi-static sensitivity calibration results of the two composites are shown in Figure 4. The relevant parameters obtained by linear fitting are shown in Table 1.

By combining the results of Figure 4 and Table 1, a nonlinear mutation point (point G) is observed for the quasi-static sensitivity of type 1-3 and type 2-2 cement-based piezoelectric composites: the stress value of type 1-3 and type $2-2$ is approximately 21.7 and $35.0 \mathrm{MPa}$, respectively. When the applied load is lower than the critical value, a good linearity is observed between the output electric displacement and stress of the sample. When the applied load is higher than the critical value, a non-linear characteristic is observed between the output electrical displacement and stress of the sample. That is, as loading increases, the rising amplitude of the output electric displacement tends to decrease or even attenuate. This may be because loading reaches the critical value associated with the electric domain switching of the piezoelectric ceramic. Owing to the electric domain deflection, the piezoelectric performance of the sample tends to reduce. In the linear range, the quasi-static sensitivity of the type 1-3 and type 2-2 cement-based piezoelectric composites is 157 and $169 \mathrm{pC} / \mathrm{N}$; the difference between the two is not obvious. However, the linear range associated with the quasi-static sensitivity of the type 2-2 is significantly larger (by 
$61.3 \%$ ) than that of type 1-3. Overall, the piezoelectric sensing performance of type 2-2 is better than that of type 1-3.

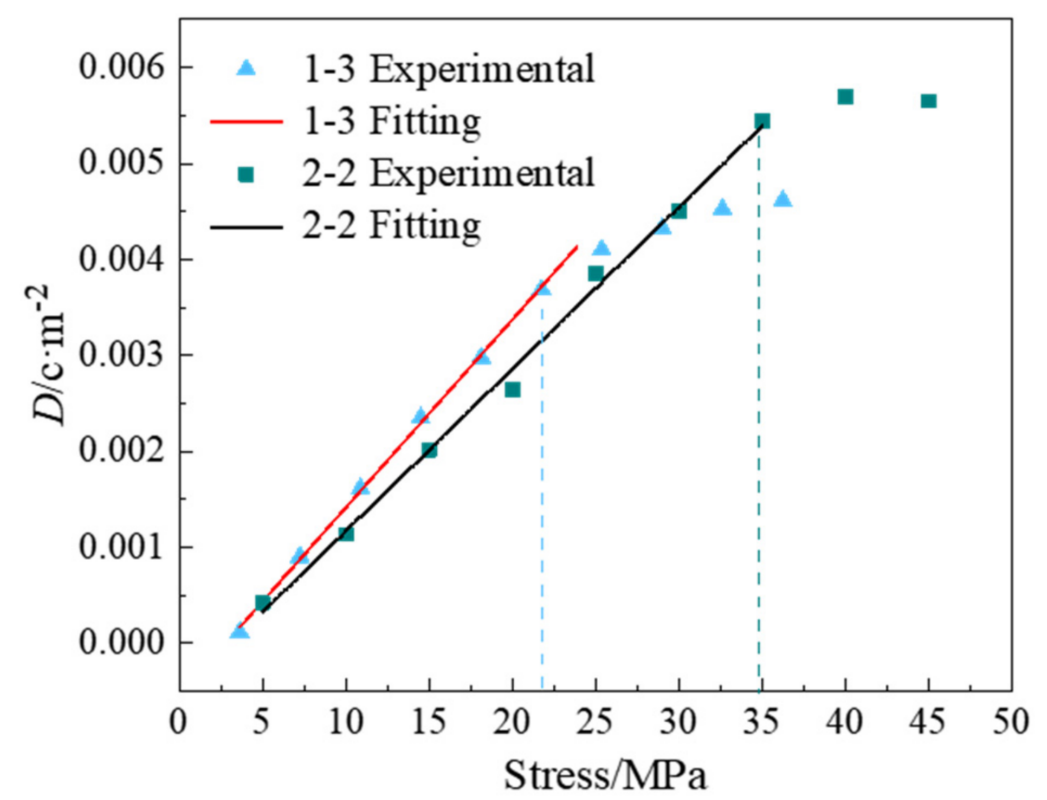

Figure 4. Calibration of quasi-static sensitivity.

Table 1. Linear fitting parameters of quasi-static sensitivity.

\begin{tabular}{cccccc}
\hline Parameter & Sample & Linearity Range/MPa & Fitting Function & $\begin{array}{c}\text { Correlation } \\
\text { Coefficient } \mathbf{R}^{\mathbf{2}}\end{array}$ & Sensitivity/(pC.N $^{-\mathbf{1})}$ \\
\hline $1-3$ & $0-21.7$ & $D=0.000157 \sigma-0.43533$ & $99.88 \%$ & 157 \\
$2-2$ & $0-35.0$ & $D=0.000169 \sigma+0.00052$ & $99.49 \%$ & 169 & \\
\hline
\end{tabular}

\subsection{Impact Compression Test}

The impact compression test was performed on the type 1-3 and type 2-2 cementbased piezoelectric composite samples, and the dynamic mechanical properties of the two samples under different strain rates were obtained. The original waveforms of the electrical signals associated with the strain gauges on the incident and transmission bars were recorded by the oscilloscope (Figure 5). In the figure, Figure 5a,b represent the waveform without and with the waveform shaper, respectively; the waveform effectively prolongs the rising time of the incident wave. The penetration depth of the bullet inside the ballistic is $300 \mathrm{~mm}$, and the strike pressure is $0.2,0.4,0.6,0.8$, and $1.0 \mathrm{MPa}$.

According to the test results, the stress-strain curves of the samples under different strain rates are obtained using Formulas (1)-(4), as shown in Figure 6. With the continuous increase in the strain rate, the stress values of type 1-3 and type 2-2 specimens increase under the same strain. This result indicated that both the cement-based piezoelectric composites exhibit evident strain rate effects and can be regarded as strain rate sensitive materials.

This study refers to the Maxwell model used by Zhang [28] to characterize the stress relaxation effect of cement-based piezoelectric composites. The stress relaxation effect of type 1-3 and type 2-2 samples under impact compression has also been characterized. The differential equation used in the Maxwell model can be expressed as Formula (5):

$$
\frac{\mathrm{d} \sigma}{d t}+\frac{\sigma}{\tau}=E_{1} \dot{\varepsilon}
$$

where $E_{1}$ is the Young's modulus of the spring, $\tau$ is the stress relaxation time of the viscous pot, and $\dot{\varepsilon}$ is the total strain rate of the model. 


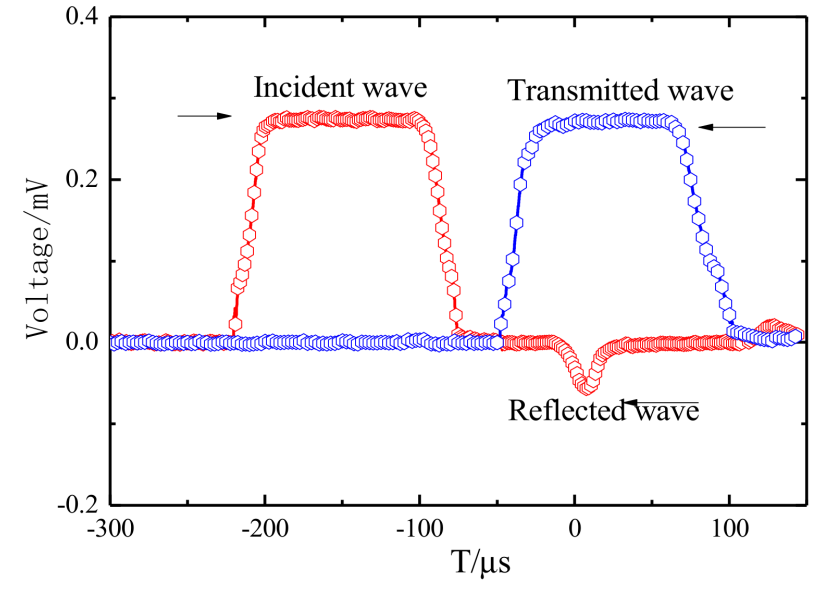

(a)

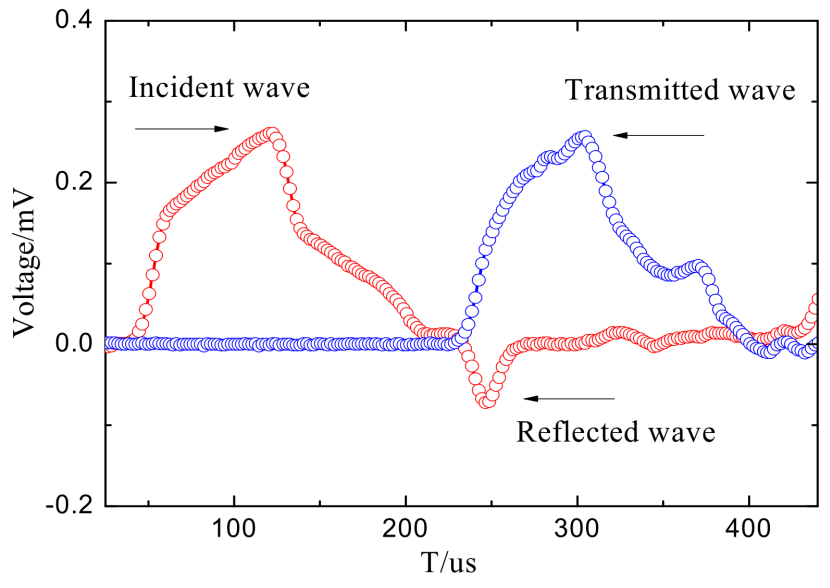

(b)

Figure 5. Original waveform of the sample under impact loading. (a) Original waveform without the waveform shaper; (b) Original waveform with waveform shaper.

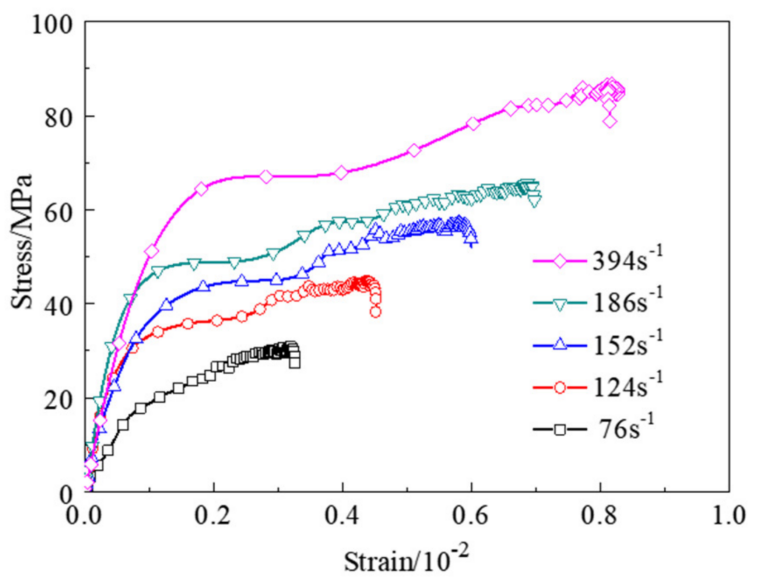

(a) 1-3

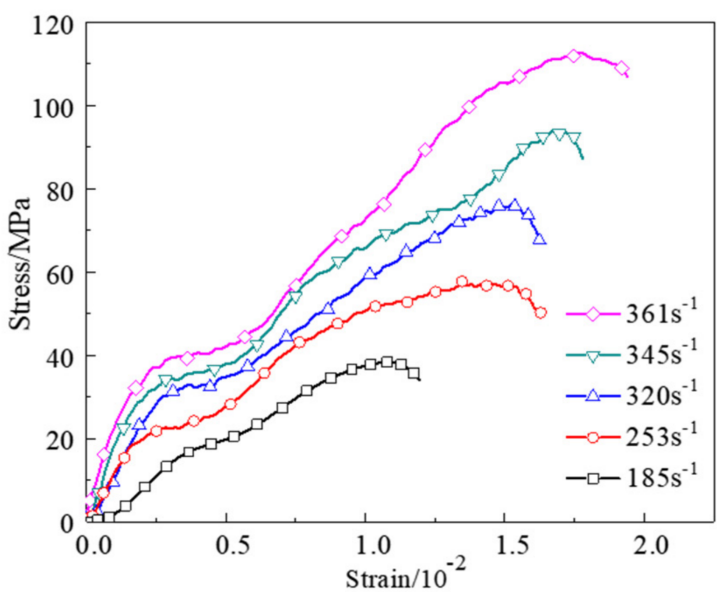

(b) 2-2

Figure 6. Stress-strain curves of the sample under different strain rates.

For the constant strain rate process, the differential equation of the Maxwell model can be expressed by Formula (6), where $\varepsilon$ is the total strain of the model.

$$
\sigma=E_{1} \tau \dot{\varepsilon}\left(1-e^{-\frac{\varepsilon}{\tau \dot{\varepsilon}}}\right)
$$

According to Formula (6), the data on the stress-strain curves of type 1-3 and type 2-2 composites under different strain rates are fitted. Figure 7 shows the stress relaxation time $\tau$ of the viscous pot under different strain rates. The stress relaxation time and strain rate show an approximately negative linear relationship, regardless of the type of cementbased piezoelectric composite; that is, the relaxation time decreases monotonously with an increase in strain rate. However, under the same strain rate, the relaxation time of type 2-2 is lower than that of type 1-3, indicating that the time required for type 2-2 to return to its normal state is lower after the external force is released. This feature is more advantageous in the application of engineering sensors. 


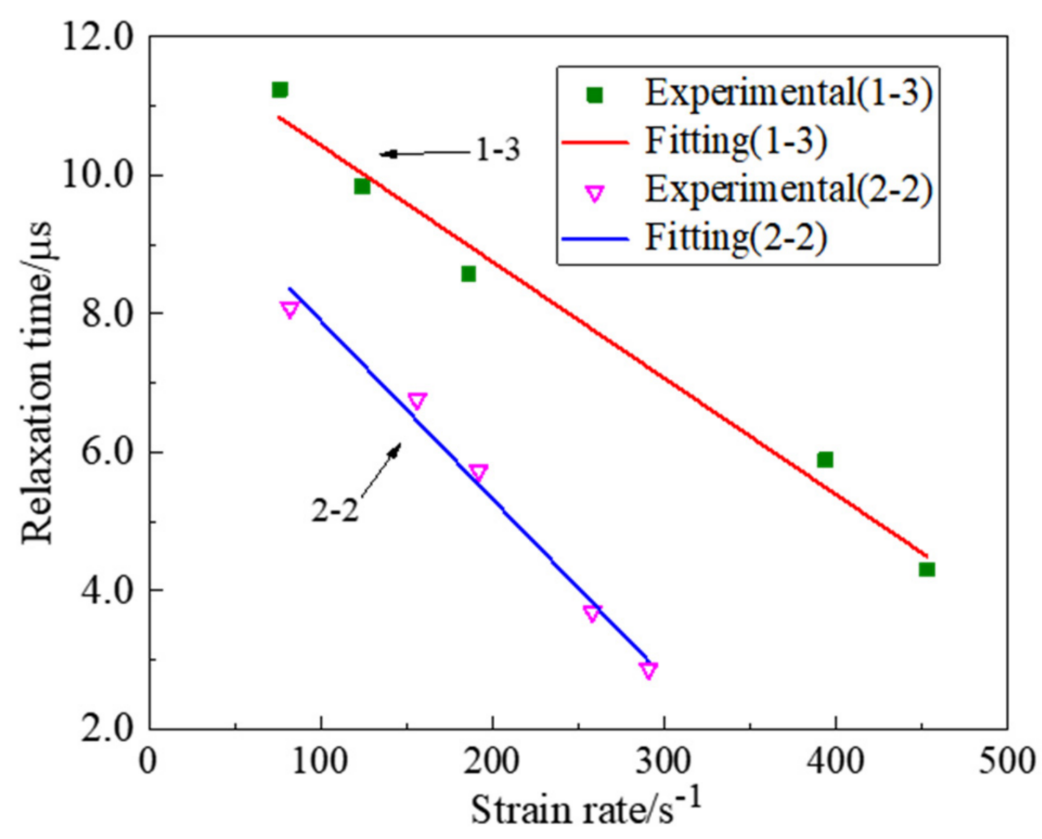

Figure 7. Relationship between relaxation time and strain rate.

\subsection{Electro-Mechanical Response Analysis}

Because the sample generates a larger voltage at the time of impact loading, a current mode circuit is used to measure the electrical signal output by the sample in order to better protect the circuit. The oscilloscope collects the voltage across the resistor in real time. The voltage recorded by the oscilloscope is $V(t)$ and the current in the circuit is $I(t)$.

The discharge at both ends of the sample is calculated as Formulas (7) and (8):

$$
Q(t)=\int_{0}^{t} I(t) d t=\int_{0}^{t} \frac{V(t)}{R} d t
$$

The electric displacement of the sample is expressed as:

$$
D(t)=\frac{Q(t)}{A_{0}}
$$

where $A_{0}$ is the cross-sectional area of the sample.

The ratio of $D(t)$ to $\sigma(t)$ represents the dynamic sensitivity $K$ of the sample.

Based on Formulas (7) and (8) and the definition of sensitivity, the stress and electric displacement time curves of type 1-3 and type 2-2 samples are shown in Figure 8. The stress-time curves of type 1-3 (Figure 8a) and type 2-2 (Figure 8b) composites are highly similar to their output electric displacement time curves, indicating that the output electric displacement of the sample can accurately respond to the impact loading of the sample; this implies that type 1-3 and type 2-2 composites are good electro-mechanical sensing materials, rendering them applicable in the production of engineering sensors.

Figure 9 shows the electric displacement-time curves of the composites under different strain rates. At lower strain rates, an increase in the loading strain rate causes the peak values of the output electric displacements corresponding to type 1-3 (Figure 9a) and type 2-2 (Figure 9b) samples to show an increasing trend. At higher strain rates, an increase in the loading strain rate causes the growth rate associated with the amplitude of output electric displacement to decrease. 


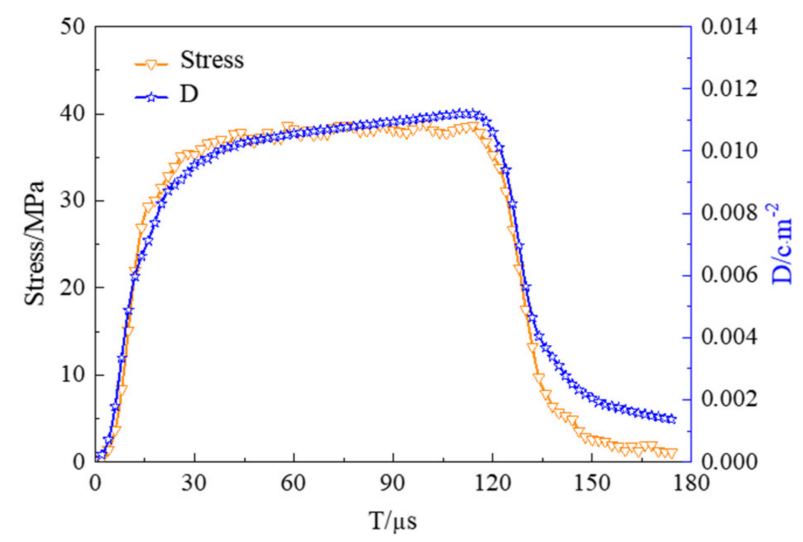

(a) 1-3

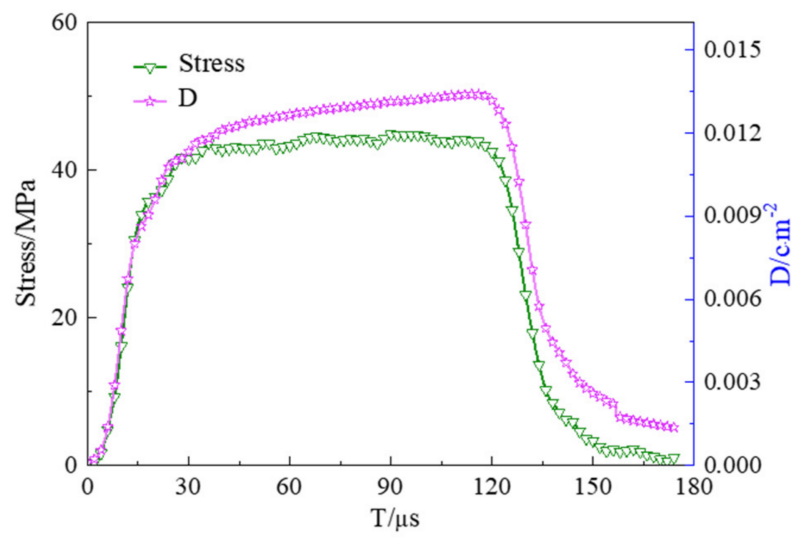

(b) $2-2$

Figure 8. Time curves of the stress and electric displacement of the composite.

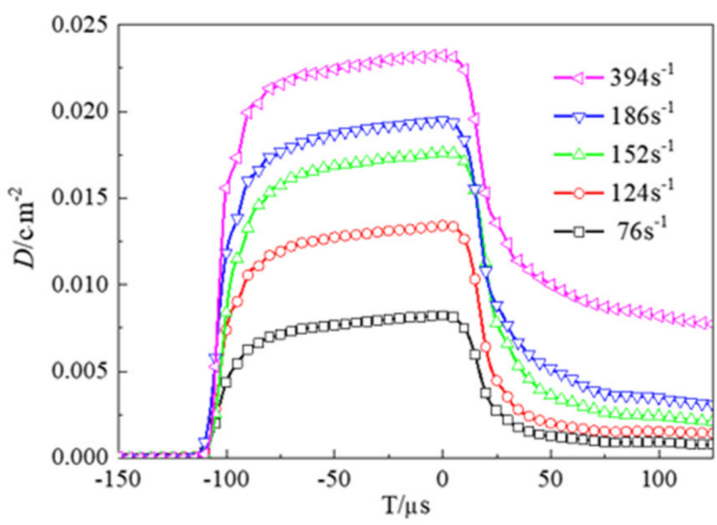

(a) 1-3

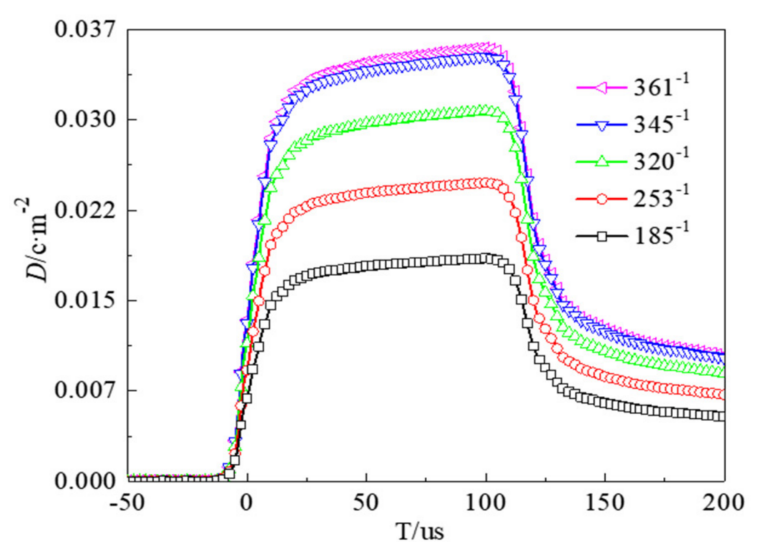

(b) 2-2

Figure 9. Electrical displacement-time curves of the composite.

Based on Figures 6, 9 and 10 shows the variation in the stress peak value and output electric displacement of the samples under different strain rates. MATLAB is used to linearly fit the test data, and the slope of the straight line represents the dynamic sensitivity of the cement-based piezoelectric composite. The relevant parameters obtained by linear fitting are shown in Table 2.

By combining Figure 10 and Table 2, results similar to those obtained under quasistatic compression are obtained; that is, the dynamic sensitivity of type 1-3 and type 2-2 composites also exhibits electrical domain switching points, namely: 70.5 and 88.0 MPa. When the applied load is less than the domain switching point (first yielding point), the electro-mechanical response of type 1-3 and type 2-2 exhibits a good linear relationship. In the linear range, the dynamic sensitivity of type 1-3 and type 2-2 is 323 and $296 \mathrm{pC} / \mathrm{N}$, respectively. When the applied load exceeds the domain switching point, the increase in the output electric displacement is attenuated. Subsequently, the output electric displacement and the stress of the sample no longer show a linear relationship because the piezoelectric ceramics eventuate domain change, which reduces the piezoelectric performance of the sample.

Overall, the difference in the sensitivity of type 1-3 and type 2-2 is observed to be minor; however, the linear range of type $2-2$ is significantly greater than that of type 1-3 (by $24.8 \%$ ). 


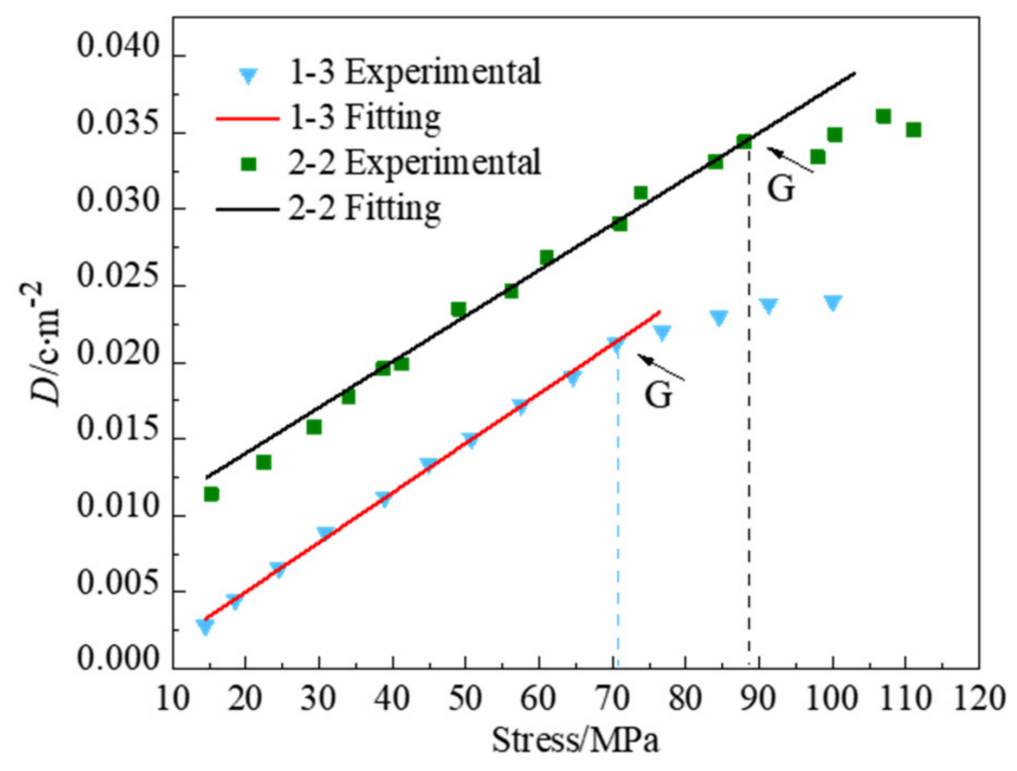

Figure 10. Calibration curve for dynamic sensitivity of the cement based piezoelectric composites.

Table 2. Linear fitting parameters of dynamic sensitivity.

\begin{tabular}{cccccc}
\hline Parameter & Sample & Linearity Range/MPa & Fitting Function & $\begin{array}{c}\text { Correlation } \\
\text { Coefficient } \mathbf{R}^{2}\end{array}$ & Sensitivity/(pC.N-1) $^{-1}$ \\
\cline { 1 - 4 } $1-3$ & $0-70.5$ & $D=0.000323 \sigma-0.0014$ & $99.81 \%$ & 323 \\
$2-2$ & $0-88.0$ & $D=0.000296 \sigma+0.0082$ & $98.35 \%$ & 296 \\
\hline
\end{tabular}

\section{Conclusions}

Using the ordinary Portland cement as the matrix and PZT-5H piezoelectric ceramics as the functional body, type 1-3 and type 2-2 cement-based piezoelectric composites were prepared in this study via the cutting-casting method. The electro-mechanical response characteristics of type 1-3 and type 2-2 under quasi-static and dynamic impact compression are studied. The following conclusions can be drawn:

(1) Under quasi-static compression, the sensitivity of type 1-3 and type 2-2 composites has a nonlinear mutation point (approximately 21.7 and $35.0 \mathrm{MPa}$, respectively). The linear range of type 2-2 is significantly greater than that of type 1-3 (by $61.3 \%$ ).

(2) Both type 1-3 and type 2-2 composites have strain rate effects and are strain rate sensitive materials. At a lower strain rate, the peak value of the output electric displacement increases as the strain rate rises; at a higher strain rate, the growth rate associated with the peak value of the output electric displacement slows down.

(3) The stress relaxation time and strain rate exhibit an approximately negative linear correlation for both type 1-3 and type 2-2; that is, the relaxation time monotonously decreases with an increase in strain rate. The time required for type 2 to return to its normal state is lower after the external force is released.

(4) Similar to the results obtained under quasi-static compression, the dynamic sensitivity of type 1-3 and type 2-2 also has electrical domain switching points (70.5 and $88.0 \mathrm{MPa}$, respectively). The linear range of type 2-2 is greater than that of type 1-3 (by 24.8\%). Therefore, type 2-2 has certain advantages as a sensor material, regardless of whether it is subjected to quasi-static or dynamic loading. 
Author Contributions: Conceptualization, Y.L. and Y.Z.; validation, J.C. and C.S.; formal analysis, Y.L.; investigation, Y.L. and H.D.; data curation, Y.L. and W.C.; writing-original draft preparation, Y.L.; writing-review and editing, Y.L.; supervision, J.C.; project administration, Y.L. and J.C. All authors have read and agreed to the published version of the manuscript.

Funding: This research was funded by Natural Science Foundation of China (Nos. 11572163 and 11832013), Ningbo Rail Transit (JS-00-SG-17003).

Institutional Review Board Statement: Not applicable.

Informed Consent Statement: Informed consent was obtained from all subjects involved in the study.

Data Availability Statement: The data used to support the findings of this study are available from the corresponding author upon request.

Acknowledgments: The authors gratefully acknowledge the financial support from the National Natural Science Foundation of China and Ningbo Rail Transit Ltd.

Conflicts of Interest: The authors declare no conflict of interest.

\section{References}

1. Tasuji, M.E. Stress-stain Response and Fracture of Concrete under Multiaxial Conditions. ACI 1978, 75, $306-312$.

2. Chen, J.K.; Jiang, M.Q.; Zhu, J. Damage evolution in cement mortar due to erosion of sulphate. Corros. Sci. 2008, 50, $2478-2483$. [CrossRef]

3. Chen, J.K.; Jiang, M.Q. Long-term evolution of delayed ettringite and gypsum in Portland cement mortars under sulfate erosion. Constr. Build. Mater. 2008, 23, 812-816. [CrossRef]

4. Spark, P.R.; Menzies, J.B. The effect of rate loading upon the static and fatigue strengths of plain concrete in compression. Mag. Concr. Res. 1973, 25, 73-801. [CrossRef]

5. Ou, J.P.; Han, B.G. Piezoresistive Cement-based Strain Sensors and Self-sensing Concrete Components. J. Intell. Mater. Syst. Struct. 2009, 20, 329-336.

6. Garcia-Macias, E.; Downey, A.; D’Alessandro, A.; Castro-Triguero, R. Enhanced lumped circuit model for smart nanocomposite cement-based sensors under dynamic compressive loading conditions. Sens. Actuators A Phys. 2017, 260, 45-57. [CrossRef]

7. Yuan, M.Z.; Zhang, J.R.; Yang, L.Z. Processing method and property study for cement-based piezoelectric composites and sensors. Mater. Res. Innov. 2015, 19, S134-S138. [CrossRef]

8. Sha, F.; Xu, D.; Huang, S. Mechanical Sensing Properties of Embedded Smart Piezoelectric Sensor for Structural Health Monitoring of Concrete. Res. Nondestruct. Eval. 2021, 32, 1-25. [CrossRef]

9. Cédric, D.; Arnaud, D. A study on the performance of piezoelectric composite materials for designing embedded transducers for concrete assessment. Smart Mater. Struct. 2018, 27, 035008.

10. Sikora, P.; Chung, S.Y. Cement-Based Composites: Advancements in Development and Characterization. Crystals 2020, 10, 832. [CrossRef]

11. Dong, B.; Xing, F.; Li, Z. Cement-Based Piezoelectric Ceramic Composite and Its Sensor Applications in Civil Engineering. ACI Mater. J. 2011, 108, 543-549.

12. Wei, Z.; Gao, J.; Shi, B.; Cui, H.; Zhu, H. Health Monitoring of Rehabilitated Concrete Bridges Using Distributed Optical Fiber Sensing. Comput. Aided Civ. Infrastruct. Eng. 2010, 21, 411-424.

13. Dong, Z.; Li, Z.; Wu, K.R. 2-2 Piezoelectric cement matrix composite: Part II. Actuator effect. Cem. Concr. Res. 2002, 32, 825-830.

14. Lee, S.J.; Ahn, D.; You, I.; Yoo, D.Y.; Kang, Y.S. Wireless cement-based sensor for self-monitoring of railway concrete infrastructures. Autom. Constr. 2020, 119, 103323. [CrossRef]

15. Chaipanich, A.; Rianyoi, R.; Potong, R.; Jaitanong, N. Aging of 0-3 piezoelectric PZT ceramic-Portland cement composites. Ceram. Int. 2014, 40, 13579-13584. [CrossRef]

16. Ding, W.J.; Xu, W.W.; Dong, Z.J.; Liu, Y.Q.; Wang, Q.; Shiotani, T. Piezoelectric properties and microstructure of ceramicrete-based piezoelectric composites. Ceram. Int. 2021, 47, 29681-29687. [CrossRef]

17. Ding, W.J.; Liu, Y.Q.; Wang, Q.; Han, N.X. Cement-Based Piezoelectric Ceramic Composites for Sensing Elements: A Comprehensive State-of-the-Art Review. Sensors 2021, 21, 3230. [CrossRef]

18. Chaipanich, A. Effect of PZT particle size on dielectric and piezoelectric properties of PZT-cement composites. Curr. Appl. Phys. 2007, 7, 574-577. [CrossRef]

19. Guan, X.C.; Liu, Y.C.; Li, H.; Ou, J.P. Study on Preparation and Performance of 1-3 Type Cement-based Piezoelectric Composite. J. Disaster Prev. Mitig. Eng. 2010, 30, 345-347. (In Chinese)

20. Sun, Y.; Deng, J.P. The influence of forming pressure on the 0-3 type PZT/cement piezoelectric composite. Acta Mater. Compos. Sin. 2017, 34, 1146-1151. (In Chinese)

21. Hou, S.; Zhang, H.B.; Ou, J.P. SA-based concrete seismic stress monitoring for high-strength concrete. Smart Mater. Struct. 2016, 25, 095041. [CrossRef] 
22. Ma, Y.; Cheng, X.; Jiang, Q.; Li, Y. A cement-based 1-3 piezoelectric composite sensor working in $\mathrm{d}_{15}$ mode for charactering the shear stress in civil engineering structure. Smart Mater. Struct. 2018, 27, 115013. [CrossRef]

23. Newnham, R.E.; Skinner, D.P.; Cross, L.E. Connectivity and Piezoelectric-Pyroelectric Composite. Mater. Res. Bull. 1978, 13, 525-536. [CrossRef]

24. Qian, L.; Zhang, F.; Xu, X.Y.; Chen, J.Y. Research on PZT5 domain switching under impact loading. J. Funct. Mater. 2017, 48, 7093-7097, 7103. (In Chinese)

25. Tang, E.L.; Li, Y.; Wang, R.Z.; Han, Y.F.; Wang, L.; Xiang, S.H.; Li, Z.B.; Gao, G.W.; Lin, X.C.; Liu, M.; et al. Research on Dynamic Response and Electrical Output Characteristics of PZT-5H Piezoelectric Ceramics under Strong Impact. Binggong Xuebao Acta Armamentarii 2018, 39, 983-990. (In Chinese)

26. Zhang, F.; Feng, P.J.; Wang, T.; Chen, J.Y. Mechanical-electric response characteristics of 1-3 cement based piezoelectric composite under impact loading. Constr. Build. Mater. 2019, 228, 116781. [CrossRef]

27. Rong, H.; Zhi, F.S. Dynamic analysis of sandwich cement-based piezoelectric composites. Compos. Sci. Technol. 2012, 72, 894-901.

28. Wang, L.L.; Wang, Y.G. The important role of stress wave in studying the dynamic constitutive properties of materials with SHPB. Explos. Shock. Waves 2005, 1, 17-25. 